\title{
Accuracy of a fourth generation apex locator-an in vitro evaluation
}

\author{
Dalia Abdullah ${ }^{1}$ and Mariam Abdullah ${ }^{2}$ \\ ${ }^{1}$ Department of Operative Dentistry Faculty of Dentistry, UKM \\ ${ }^{2}$ Department of Conservative Dentistry, Faculty of Dentistry, UM \\ Kuala Lumpur
}

\begin{abstract}
The new fourth generation electronic apex locator (EAL), Elements (SybronEndo, USA) has been introduced recently in the market. This study aims to investigate the accuracy of this EAL and to compare the result with a well-known apex locator, Root ZX and the radiographic technique using an in vitro model. Thirty anterior teeth with straight canals stored in $10 \%$ formalin were used. Access cavities were prepared followed by coronal flaring of the canals. Water was used as an irrigant. After the actual lengths (AL) were measured, the teeth were then embedded in an alginate model. Periapical radiograph of each tooth was taken using a digital sensor and the radiographic lengths $(R L)$ were measured $0.5 \mathrm{~mm}$ short of the radiographic apex. Electronic tooth length measurements (EL) were carried out using both EAL. Canals were then irrigated with $2.5 \% \mathrm{NaOCl}$ and EL was taken again. Results showed that both EALs were highly accurate to within $+0.5 \mathrm{~mm}$ of the apical foramen, with mean differences between the AL and EL of Elements $0.23 \mathrm{~mm}$ $(S E=0.04)$ and Root $Z X$ was $0.31 \mathrm{~mm}(S E=0.05)$. RL was significantly less accurate compared to the readings from both EAL. No significant difference was found in the reading between both apex locators when measurements were taken in NaOCl solution. Both Elements and Propex proved to be as reliable as Root ZX. Presence of sodium hypochlorite solution did not affect the accuracy of the measurements.
\end{abstract}

Key words: electronic apex locator, elements, root ZX, alginate model

Correspondence: Dalia Abdullah, Department of Operative Dentistry, Faculty of Dentistry, UKM. Jalan Raja Muda Abdul Aziz, 50300 Kuala Lumpur. Email: daliaabd@medic.ukm.my/daliaabd@ hotmail.com; Tel: 034040 5769, Fax: 0340405794.

\section{INTRODUCTION}

The objective of root canal treatment is to provide an environment that allows healing of periradicular tissues. One of the important steps to achieve this objective is to clean and shape the entire canal system from the orifice to the minor apical foramen. This entire length of canal is commonly called as the "working length". Before the development of electronic apex locators, working length was determined by measuring tooth length in pre-operative radiographs. Kuttler ${ }^{1}$ in his study reported that the minor apical foramen is commonly located about $0.5-1 \mathrm{~mm}$ from the anatomical apex. Based on this fact, the common practice of estimating working length was to measure the length of the root from the radiographic apex to the crown and then deducting $0.5-1 \mathrm{~mm}$ from the length. However, this method has been reported to be unreliable and inaccurate as the radiographic images, most of the time was found to be distorted. ${ }^{2}$

Electronic apex locator (EAL) is an important adjunct in root canal treatment. It helps to locate the minor apical foramen and therefore reduces the error during working length determination. Sunada ${ }^{3}$ introduced the first apex locator after he discovered that he could use the constant value of impedance of both periodontal ligament and the oral mucosa to locate the position of the apical foramen. The earlier two generations of apex locators however, tend to give poor readings and its performance could be influenced by canal contents such as presence of pulp tissues and irrigants. ${ }^{4}$ Since then, improvements were made to the device that led to the development of apex locators using multiple frequencies to increase the accuracy. One of the third generation apex locators, Root ZX (J. Morita, Tokyo, Japan), had been extensively studied and was reported to be $95-100 \%$ accurate. ${ }^{5,6}$ It was proven to be reliable even in the presence of body fluid, pulp tissues and irrigants. ${ }^{7}$ In 2003, Elements Diagnostic Unit (SybronEndo, Anaheim, USA), a fourth generation EAL was newly introduced in the market. It is a 2-in-1 unit with the second function of vitality testing. To determine the length, it uses the principle of measuring the impedance value by calculating both the resistance and capacitance and comparing this value with the database built in the device. This technique of measurement was claimed by manufacturer to increase the accuracy and reliability of the device. ${ }^{8}$ The purpose of this study were, to investigate the accuracy of Elements EAL using an in vitro model, to compare its accuracy with Root $\mathrm{ZX}$ and to the length obtained from radiographs, and to investigate its accuracy of measurement in the presence of $2.5 \%$ sodium hypochlorite $(\mathrm{NaOCl})$ solution. 


\section{MATERIALS AND METHODS}

Thirty single-rooted anterior extracted teeth were collected from various dental clinics and kept wet in $10 \%$ formalin. These were mature teeth with completely formed apices and straight roots with the absence of apical resorption. Presence of a patent apical canal was confirmed by allowing a \#6 file to protrude through the apical foramen. The incisal edges were trimmed and leveled with a diamond bur to provide fixed coronal reference points to get accurate and reproducible measurements. Standard endodontic access cavities were made at the palatal surfaces. Canals were flared coronally using Gates Glidden drills (Dentsply, Switzeland) size 2 and 3. Canals were irrigated with water using a $3 \mathrm{ml}$ syringe and $25 \mathrm{G}$ hypodermic needle.

Determination of actual length (AL): AL was measured by introducing a size $15 \mathrm{~K}$-file (Dentsply, Switzeland) into each canal until the tip of the file was just visible at the main foramen using the microscope OMPI PICO (Carl Zeiss, Germany) under $2.5 \times$ magnification (from 0, 45 and 90 degree angle). Each measurement was repeated 3 times and an average was computed. Alginate model specially developed by Kaufmann et al. ${ }^{9}$ for testing apex locators was used in this study. The model consisted of teeth embedded in alginate in a plastic box (Figure 1). The alginate model was kept moist in the refrigerator when not in use throughout the experiment. Pink wax was used to block an area next to the embedded teeth to accommodate placement of the radiographic digital sensor for taking radiographs.

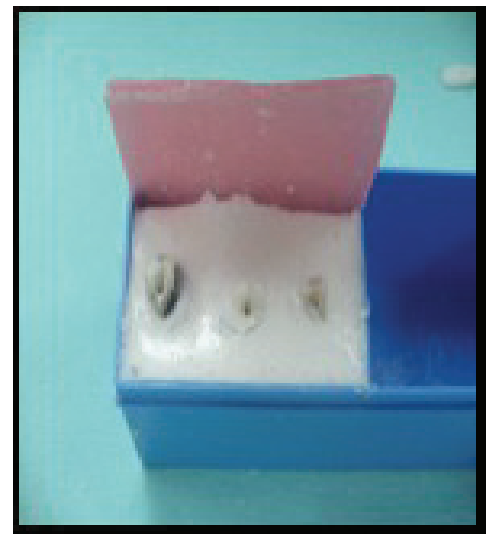

Figure 1. Alginate model.

Determination of radiographic length (RL): Radiovisiograph ( $\mathrm{RVG}$, Trophy) was used to take the images of the teeth. The RVG sensor was placed in the allocated slot, which was parallel vertically and horizontally to the teeth. The sensor was placed as near as possible to the teeth, distance from the sensor and x-ray cone was fixed at $4 \mathrm{~cm}$ and with the exposure time fixed at $0.17 \mathrm{~s}$. Software Trophy Windows Ver 5.04 was used to visualize the images. The tooth length was calibrated, measured and recorded under $100 \%$ magnified images by measuring the coronal reference point to the radiographic apex. A $0.5 \mathrm{~mm}$ was deducted from this length to give the RL as what was usually practiced clinically.

Determination of electronic length (EL): Lip clip was attached to alginate and the file clip was attached to the file in each tooth. Measurement was taken with K-files of appropriate sizes (first file that binds to the apex). For Root ZX, measurement of the length was taken when the device indicated 'Apex'. For Elements (Figure 2), measurement was taken when reading was at ' 0 ' (although the instructional manual stated that from ' 0 ' reading, the file should be withdrawn to $0.5 \mathrm{~mm}$ mark to achieve working length clinically). Measurements were repeated 3 times for each tooth and the third working length measurement was taken for analysis.

Determination of electronic length (EL) in the presence of $2.5 \% \mathrm{NaOCl}$ : All canals were irrigated with $15 \mathrm{ml} 2.5 \%$ $\mathrm{NaOCl}$ solution. Excess solution in the canal was aspirated using the irrigating syringe and EL was taken again using both EALs. To prevent bias, measurement for all 30 teeth was done at a random order.

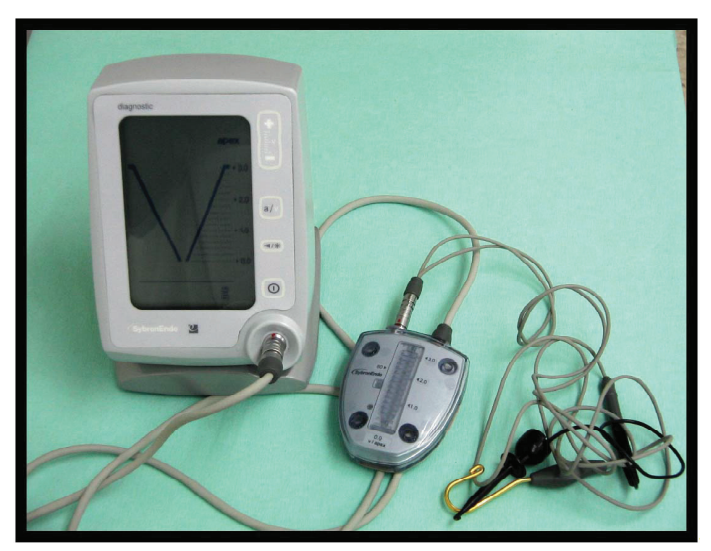

Figure 2. Elements apex locator.

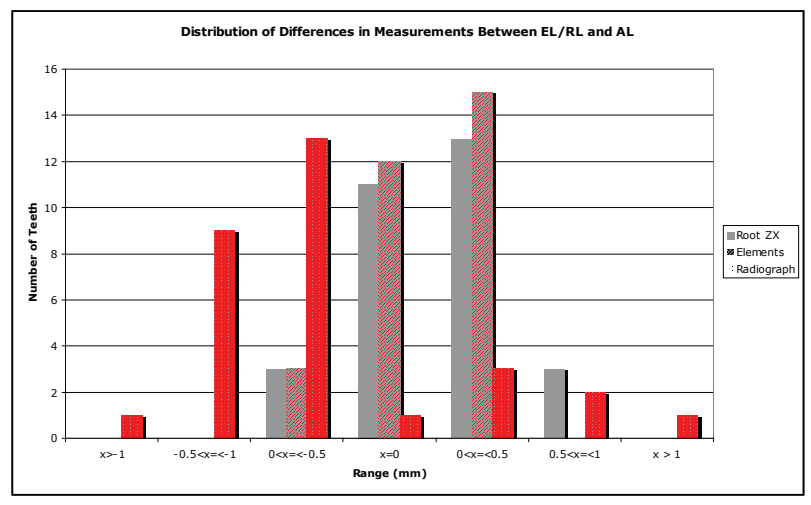

Figure 3. Distribution of the difference between RL and EL with AL $(\mathrm{N}=30)$.

Data analysis: All the measurements of RL and EL were deducted from the AL for each tooth to give a value of the difference. The negative value indicated the length was short of the apex, zero indicated that length was at the apex and positive value indicated that the length went 
beyond the apex. Mean difference of RL and EL (for all 30 teeth) were computed. Results were subjected to statistical analysis (Paired T-test and ANOVA with repeated measurements). Statistical significance was accepted at $\mathrm{p}$ value of less than 0.05 .

\section{RESULTS}

Water as irrigant-Measurement obtained from Elements (Table 1) was found to be closer to AL with the mean difference of $0.23 \mathrm{~mm}$. However, the result was not statistically significant as the mean value of measurement taken from Root ZX EAL was at $0.31 \mathrm{~mm}(\mathrm{p}=0.108)$. The RL was significantly less accurate compared to the readings taken from both EALs with mean difference of $0.60 \mathrm{~mm}$ with the $p=0.00$ for Element and $p=0.002$ for Root ZX.

$\mathrm{NaOCl}$ as irrigant-Mean difference of EL taken with both Elements and Root $\mathrm{ZX}$ in the presence of $\mathrm{NaOCl}$ solution were shown in Table 1. Statistical analysis of mean difference using ANOVA (post hoc test/Turkey's method) showed no significant difference between the reading of both EALs taken with and without the presence of $\mathrm{NaOCl}(P=1)$.

Difference of EL with AL for all teeth were categorized into 7 groups according to distance from apical foramen (Figure 3).

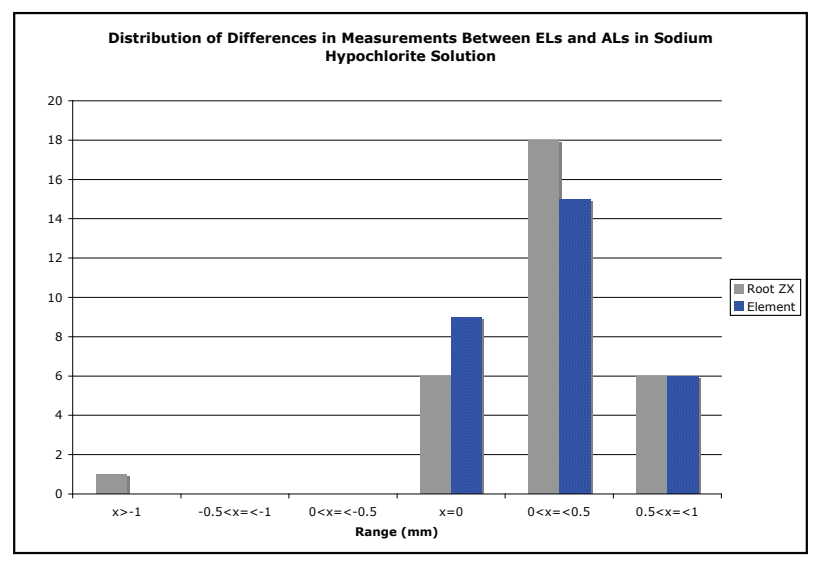

Figure 4. Distribution of differences in measurement between EL and AL in $2.5 \%$ sodium hypochlorite.

Water as irrigant-Ninety percent $(n=27)$ of the EL readings taken from Elements fell within the range of 0 or $0.5 \mathrm{~mm}$ shorter than $\mathrm{AL}$, for Root $\mathrm{ZX}$ it was $80 \%$ $(n=24)$, whilst only $46 \%(n=4)$ of the readings taken from radiographs fell in within this range. In comparison,
$43 \%$ of the $\mathrm{RL}$ readings were about $0.5 \mathrm{~mm}$ longer than the actual length (Figure 3).

$\mathrm{NaOCl}$ as irrigant-Eighty percent $(n=24)$ of the EL readings taken from both Elements and Root ZX fell within the range of 0 or $0.5 \mathrm{~mm}$ shorter than AL (Figure 4).

\section{DISCUSSION}

The results obtained from this study indicate that Elements apex locator was able to locate the minor apical diameter $0-0.5 \mathrm{~mm}$ short of the root length, and its performance was at par with Root ZX.

The mean values of both devices were below $0.5 \mathrm{~mm}$ with no statistical significant difference and both values were shorter than actual length. This apical position of less than $0.5 \mathrm{~mm}$ from the root end could be considered clinically acceptable as the distance of the apical constriction to the major apical foramen (end of the root) is approximately $0.5-0.8 \mathrm{~mm}$ in all tooth types. ${ }^{1}$ Apical constriction represents the boundary between the pulpal and periodontal tissue with the smallest diameter of the canal (minor apical foramen). This location was considered to be an ideal position to end the preparation and obturation of root canals, as irritation to the periodontal tissues (by irrigants, obturating materials) was kept minimal (creating a small wound site) and therefore would provide an optimal condition for healing of the periapical tissues. ${ }^{10}$ Even epidemiological study had shown that the best prognosis is when the root filling lies within $2 \mathrm{~mm}$ of the apex. ${ }^{11}$ However, determining the location of this boundary could not be done accurately by using the radiographs. Modern apex locators, as shown in this study have the ability to determine this position with greater accuracy.

Root ZX uses two frequencies to determine distance from the end of the apex, which accounts for its accuracy. ${ }^{12}$ The results from our study showed that $80 \%$ of the 30 readings fell within $0.5 \mathrm{~mm}$ and the percentage increased to $90 \%$ if $1.0 \mathrm{~mm}$ distance from the apex was taken as a reference point. These readings were reproducible even when taken in the presence of sodium hypochlorite.

Elements apex locator showed similar result in which $90 \%$ of the 30 readings fell within $0.5 \mathrm{~mm}$ from the apex. The readings were reproducible when taken in the presence of sodium hypochlorite with no significant difference in the mean value. The manufacturer claimed that its consistent readings were attributed mainly to its mechanical measurement whereby a composite waveform of two signals, 0.5 and $4 \mathrm{kHz}$ were used. These signals go through a digital-to-analogue converter to be converted into

Table 1. Mean differences between RL, EL with AL $(\mathrm{N}=30)$

\begin{tabular}{llcc}
\hline \multicolumn{1}{c}{ Solution used for irrigation } & Root ZX (SE) & Elements (SE) & Radiograph (SE) \\
\hline Water & $031(0.50)$ & $0.23(0.03)$ & $0.60(0.60)$ \\
$2.5 \% \mathrm{NaOCI}$ & $0.48(0.14)$ & $0.43(0.13)$ & - \\
\hline
\end{tabular}


an analogue signal, which then goes through amplification and then to the patient circuit model. The feedback signal waveforms are then fed into a noise reduction circuit to give the reading. ${ }^{8}$ One point to note is that a smaller percentage of readings (10-20\%) from both EAL were $0.5 \mathrm{~mm}$ longer than the actual length. Verification of the electronic measurements by radiographs may indeed be necessary to avoid trauma to the periapical tissues. During the study, it was observed that the readings from both apex locators tend to fluctuate when the canals were flooded with the solution and both devices performed much better when the excess solution was aspirated from the canal.

Radiographic method has been known to be unreliable in accurately determining working length. The measurements obtained from the radiographs were constantly longer than the actual length (mean difference of $0.60 \mathrm{~mm}$ ) and longer compared to the readings from both EALSs. These differences were statistically significant. This result was similar with Stein \& Corcoran, ${ }^{2}$ who found a difference of $0.7 \mathrm{~mm}$ between the real file positions to the measurements calculated on radiographs.

The alginate model proved to be a good and easy-tomake method to test the accuracy of apex locators in vitro. The electronic length was taken only after coronal flaring was carried on all the teeth to simulate the clinical practice. Early coronal flaring will ensure no change in length occurs after canal preparation. During the study, unstable readings were observed when a small file (eg file \#10) was used in a relatively bigger canal. A bigger file (the first file that engaged the canal wall at the apical end) was therefore used to take the measurement.

In conclusion, this study shows that Elements EAL is able to measure canal length within a clinically acceptable range and its accuracy is comparable to Root ZX. Presence of sodium hypochlorite does not affect the accuracy of the measurement. Radiographic measurements, at most time, results in overestimation of the actual length.

\section{REFERENCES}

1. Kuttler Y. Microscopic investigation of root apexes. J Am Dent Assoc $1955 ;$ 50:544-52.

2. Stein TJ, Corcoran JF. Radiographic working length revisited. Oral Surg Oral Med Oral Pathol 1992; 74:796-800.

3. Sunada I. New method for measuring the length of the root canal. J Dent Res 1962; 41:375-87.

4. Fouad AF, Krell KV, McKendry DJ, Koorbusch GF, Olson RA. Clinical evaluation of five electronic root canal length measuring instruments. J Endod 1990; 16: 446-9.

5. McDonald NJ, Pileggi R, Glickman G, Varella C. An in vivo evaluation of third generation apex locators [abstract]. J Dent Res 1999; 78:373.

6. Vajrabhaya L, Tepmongkol P. Accuracy of apex locator. Endod Dent Traumatol 1997; 13:180-2.

7. Shabahang S, Goon WW, Gluskin AH. An in vivo evaluation of Root ZX electronic apex locator. J Endod 1996; 22:616-8.

8. Gordon MPJ, Chandler NP. Electronic apex locators. Int Endod J 2004; 37:425-37.

9. Kaufman AY, Keila S, Yoshpe M. Accuracy of a new apex locator: an in vitro study. Int Endod J 2002; 35:186-92.

10. Wu MK, Wesselink PR, Walton RE. Apical terminus location of root canal treatment procedures. Oral Surg Oral Med Oral Path Oral Radiol Endod 2000; 89:99-03.

11. Sjogren U, Hagglund B, Sundqvist G, Wing K. Factors affecting the long-term results of endodontic treatment. J Endod 1990; 16:498-504.

12. Kobayashi C, Okiji T, Kaqwashima N, Suda H, Sunada I. A basic study on the electronic root canal length measurement: Part 3. Newly designed electronic root canal length measuring device using division method. Japanese Journal of Conservative Dentistry 1991; $34: 1442-8$ 